African Americans, respectively) may contribute to the absence of a survival effect of CCR2 genotypes seen in Table 1. Aiternatively, the results may reflect the relatively shorter period of follow-up, because the ALIVE began enrollment in 1988 (33). In support of the latter explanation is the elevated CCR2- $+/ 64$ "protective" genotype frequency among ALIVE slow progressors relative to rapid progressors to AIDS for the three AIDS endpoints (Fig. 2). Because this result is not statistically significant, the conclusion remains tentative until longer follow-up of African American cohorts becomes available.

45. Because the four cohorts show no significant differences in CCR2 allele or genotype frequency, they were pooled to test for significant differences between rapid and slow or nonprogressors, which were apparent. In addition, CCR2-64/ containing genotypes were higher in all cohorts for 24 of $24 \mathrm{com}$ parisons (two genotypes, four cohorts, three AIDS outcomes). Because these comparisons are interde- pendent, we applied a sign test to eight comparisons (four cohorts, two genotypes) to detect $P \leq 0.004$

46. When a conservative sign test to three genotypes and three cohorts was applied for only one outcome, there was a significant excess of $[+/ \Delta 32],[64 / /+]$, and $[64 l / 64 l]$ \{Fig. 2 and $(54)$ for $[64 l / 64 I]\}(P=$ $0.002)$

47. M. L. Levin, Acta Unio Int. Contra Cancrum 9, 531 (1953). Estimates of attributable risk were computed for Caucasians (Fig. 3); they likely vary among ethnic groups, particularly because of the differences in CCR5- $\Delta 32$ frequencies in Asians and African Amercans (18-22).

48. S. Rana et al., J. Virol. 71, $3219(1997)$

49. J. C. Stephens et al., in preparation.

50. C. C. Bleul et al., Proc. Natl. Acad. Sci. U.S.A. 94, 1925 (1997); S. Qin et al., J. Immunol. 26, 640 (1996)

51. J. Rucker et al., Cell 87, 437 (1996).

52. A. Ben-Barauch, D. F. Michiel, J. J. Oppenheim,
J. Biol. Chem. 270, 11703 (1995); O. M. Z. Howard et al., Trends Biotechnol. 14, 46 (1996); J. M. Wang and $J$. Oppenheim et al., personal communication. 53. L. Wu et al., J. Exp. Med. 185, 1681 (1997).

54. M. W. Smith et al., data not shown.

55. We gratefully acknowledge the patients, their families, and clinicians who have participated in the ALIVE, MACS, MHCS, HGDS, and SFCC cohort studies. We thank R. Boaze, R. Byrd, S. Cevario, B. Gerrard, P L loyd, M. McNally, M Malasky, S. Shrestha, E. Topper, and M. Weedon for technical assistance, and S. Edelstein, A. Munoz, S. Donfield, E. Gomperts, J. Giorgi, and J. Oppenheim for helpful discussions. The Frederick Biomedical Supercomputing Center provided computational resources used in some of our analyses. Additiona! analyses and data mentioned here can be inspected at rex.nci.nih.gov/RESEARCH/basic/lgd/front_page.htm.

9 May 1997; accepted 25 June 1997

\title{
AIB1, a Steroid Receptor Coactivator Amplified in Breast and Ovarian Cancer
}

\author{
Sarah L. Anzick, Juha Kononen, Robert L. Walker, \\ David O. Azorsa, Minna M. Tanner, Xin-Yuan Guan, \\ Guido Sauter, Olli-P. Kallioniemi, Jeffrey M. Trent, \\ Paul S. Meltzer ${ }^{\star}$
}

\begin{abstract}
Members of the recently recognized SRC-1 family of transcriptional coactivators interact with steroid hormone receptors to enhance ligand-dependent transcription. AIB1, a member of the SRC-1 family, was cloned during a search on the long arm of chromosome 20 for genes whose expression and copy number were elevated in human breast cancers. AIB1 amplification and overexpression were observed in four of five estrogen receptor-positive breast and ovarian cancer cell lines. Subsequent evaluation of 105 unselected specimens of primary breast cancer found AIB1 amplification in approximately 10 percent and high expression in 64 percent of the primary tumors analyzed. AlB1 protein interacted with estrogen receptors in a ligand-dependent fashion, and transfection of AIB1 resulted in enhancement of estrogen-dependent transcription. These observations identify AIB1 as a nuclear receptor coactivator whose altered expression may contribute to development of steroid-dependent cancers.
\end{abstract}

Gene amplification is a frequent mechanism of increased gene expression in human cancers. In breast cancer, commonly amplified chromosomal regions are derived from $17 \mathrm{q} 12,8 \mathrm{q} 24$, and $11 \mathrm{q} 13$ and encode erbB-2, c-myc, and cyclin D1, respectively (1). Molecular cytogenetic studies of breast cancers have revealed the occurrence of additional regions of increased DNA copy number whose target genes are unknown, including 20 q (2). Recently, we used chromosome microdissection and hybrid selection to clone

S. L. Anzick, J. Kononen, R. L. Walker, D. D. Azorsa, X.-Y. Guan, O.-P. Kallioniemi, J. M. Trent, P. S. Meltzer, Laboratory of Cancer Genetics, National Human Genome Research Institute, National Institutes of Health, Bethesda, MD, USA

M. M. Tanner, Laboratory of Cancer Genetics, Institute of Medical Technology, University of Tampere and Tampere University Hospital, Post Office Box 607, FIN-33101 Tampere, Finland.

G. Sauter, Institute for Pathology, University of Basel, Schönbeinstrasse 404003 Basel, Switzerland.

*To whom correspondence should be addressed. E-mail: pmeltzer@nhgri.nih.gov expressed sequences from $20 \mathrm{q}$ in an attempt to identify genes of biological significance (3). In this fashion, we isolated partial $\mathrm{cD}$ NAs for a candidate target gene termed AIB1 (amplified in breast cancer-1), which was ubiquitously expressed in normal human tissues (3). We now report that AIB1 is a member of the SRC-1 family of nuclear receptor (NR) coactivators, that it is amplified and overexpressed in breast and ovarian cancer cell lines as well as in breast cancer biopsies, that it interacts with estrogen receptor (ER), and that it functions to enhance ER-dependent transcription.

Sequence analysis of partial AIB1 CDNAs provided the first evidence of similarity between AIB1 and the SRC-1 family. SRC-1 and TIF2 are closely related transcriptional coactivators recently isolated on the basis of their affinity for $\operatorname{NRs}(4,5)$. Although the mechanism of action of SRC-1 has not been completely elucidated, in addition to interacting with NRs, SRC-1 binds to the transcriptional integrators CREB binding protein (CBP) and the closely related p300, which interact directly with the basal transcription machinery (6).

To further characterize AIB1, the fulllength cDNA was cloned and sequenced (7), revealing an open reading frame that encodes a protein of 1420 amino acids with a predicted molecular mass of $155 \mathrm{kD}$ (Fig. 1). Database searches with BLASTP identified a highly significant similarity of AIB1 with TIF2 (45\% amino acid identity) and SRC-1 (33\% amino acid identity) (8). Like TIF2 and SRC-1, AIB1 contains a basic helix-loop-helix (bHLH) domain preceding a PAS (Per/Arnt/Sim) region, serine- and threonine-rich regions, and a charged cluster. There is also a glutamine-rich region that, unlike SRC-1 and TIF2, contains a polyglutamine tract. AIB1 also contains three copies of the conserved LXXLL motif ( $\mathrm{L}=$ leucine, $\mathrm{X}=$ any amino acid), which was recently demonstrated to be critical to the coactivator receptor interaction $(9,10)$.

Because of this strong sequence similarity, we evaluated the amplification and expression of AIB1 in a series of ER-positive and -negative breast and ovarian cancer cell lines (11). AIB1 gene copy number was determined by fluorescence in situ hybridization (FISH) (Fig. 2). High-level amplification of AIB1 ( $>20$-fold) was observed in three ER-positive breast carcinoma cell lines (BT-474, MCF-7, and ZR75-1) and in one ovarian carcinoma cell line (BG-1) (Fig. 2, A and B). Overall, AIB1 was amplified in four of five ER-positive cell lines tested and in zero of six ER-negacive cell lines (12). To determine whether AIB1 amplification also occurred in uncultured cells from tumor biopsies, we screened 105 unselected breast cancer specimens for AIB1 amplification by FISH. Ten specimens of primary tumors $(9.5 \%)$ demonstrated amplification of AIB1, although the amplification levels were not as high as in the cell lines (13).

Previous interphase FISH studies have 
demonstrated that amplification of chromosome $20 \mathrm{q}$ in breast cancer is complex and involves several distinct variably coamplified chromosomal segments derived from 20q11, $20 \mathrm{q} 12$, and 20q13 (14). Importantly, in cancer cell lines BG-1 and ZR75-1, amplification of AIB1 (which maps to 20q12) has occurred independently of both the $20 \mathrm{q} 11$ and 20q13 regions (12). A similar pattern of amplification with higher copy number at AIB1 than elsewhere on $20 \mathrm{q}$ was also found in two tumor specimens (Fig. 2C). Although most instances of AIB1 amplification were observed in conjunction with increased $20 \mathrm{q} 13$ copy number, cases of $20 \mathrm{q} 13$ amplification with normal AIB1 copy number also were identified. These results indicate that AIB1 defines an independently selected region of amplification on $20 \mathrm{q}$ that takes part in a complex process of gene amplification involving multiple target regions on the same arm of the chromosome.

AIB1 expression was examined first in tumor cell lines with and without AIB1 amplification and compared with expression of ER, SRC-1, and TIF2 by Northern blotting. In direct concordance with its amplification status, AIB1 was highly overexpressed in the four ER-positive cell lines with increased AIB1 copy number (BT-474, MCF-7, ZR75-1, and BG-1) (Fig. 3A). In contrast, expression of TIF2 and SRC-1 remained relatively constant in all cell lines tested (Fig. 3A). AIB1 expression was then examined in primary breast malignancies by mRNA in situ hybridization to 75 of the tumor specimens previously used for FISH analysis (15). Normal mammary epithelium expressed moderate amounts of AIB1 mRNA (Fig. 3B). Among the tumors, high levels of AIB1 expression were observed in all 10 cases with AIB1 amplification (Fig.

3B). In addition, AIB1 expression was increased relative to normal mammary epithelium in 38 of $65(58 \%)$ of the remaining tumors or $64 \%$ overall. This observation indicates that overexpression of AIB1 by
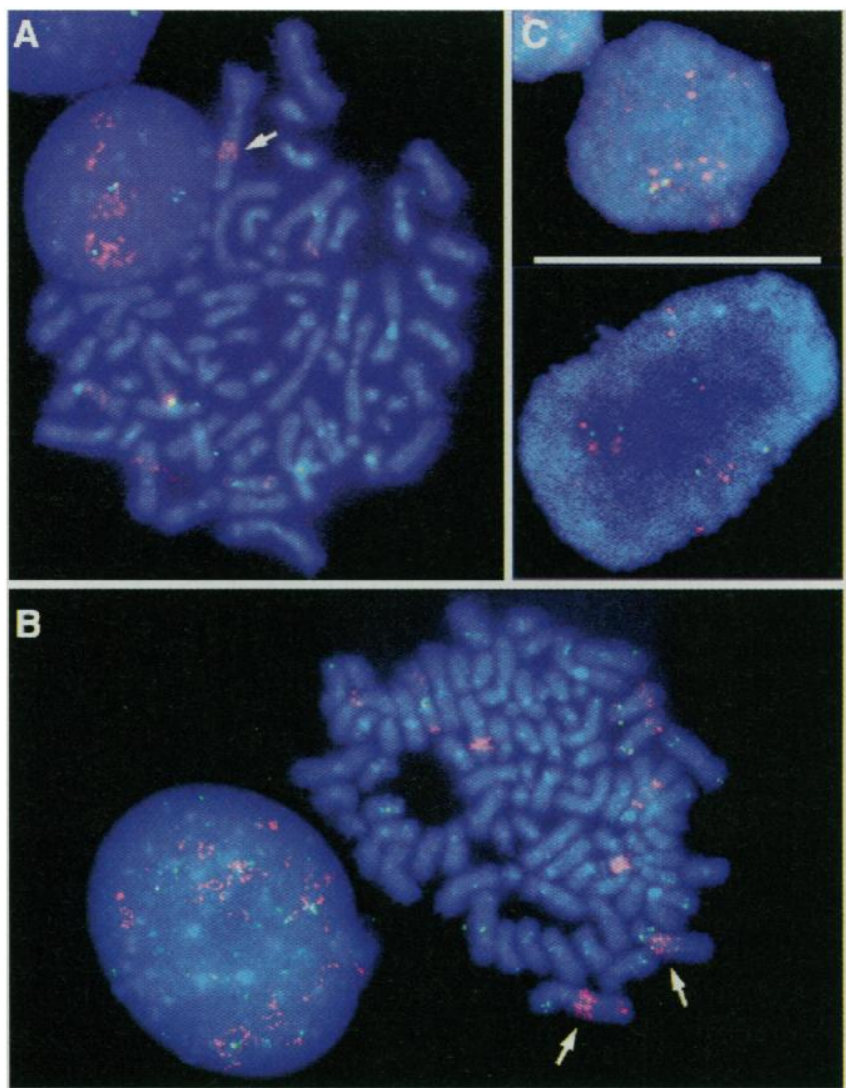

Fig. 2. Bicolor FISH analysis demonstrates AIB1 amplification (red signals) in breast cancer cell line ZR75-1 (A), ovarian cancer cell line BG-1 (B), and two uncultured breast cancer samples (C). Intrachromosomal amplification of AIB1 (arrows) is apparent in metaphase chromosomes of ZR75-1 and $B G 1$, and numerous copies of AlB1 are resolved in the adjacent interphase nuclei. The Spectrum Orange ( $V y$ sis)-labeled AlB1 P1 probe (3) was hybridized with a biotinylated reference probe for 20q11 (RMC2OP037) (A and $\mathrm{B}$ ) or a fluorescein-labeled probe for 20p (RMC20C039) (C), which appear green.
Fig. 1. Deduced amino acid sequence and structural motifs of AIB1 (GenBank accession number AFO12108) (22). Residues highlighted in black are identical in AIB1, TIF2, and SRC-1; those identical with TIF2 (GenBank accession number X97674) or SRC-1 (GenBank accession number U59302) are highlighted in gray or boxed, respectively. Three copies of the LXXLL NR interaction motif are underlined. Structural features of AIB1 include bHLH and PAS domains (with the highly conserved PAS A and $B$ regions in dark gray), serine- and threoninerich regions, and a group of charged residues (+/-). The glutamine-rich region and the polyglutamine tract are also indicated. The alignment was generated with DNASTAR software.

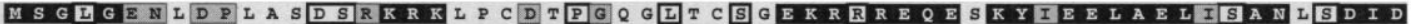

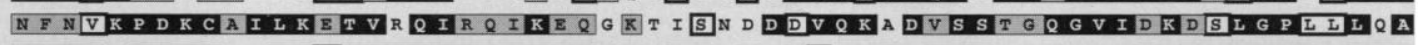

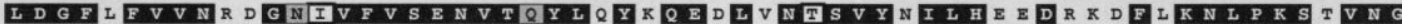

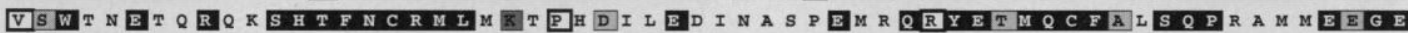

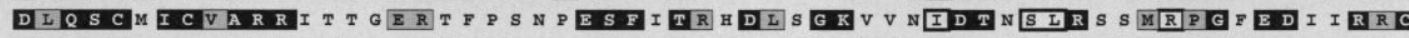

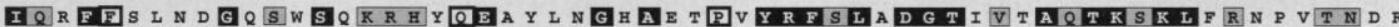

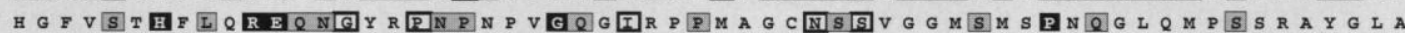

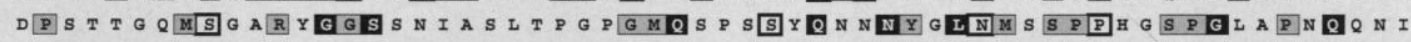

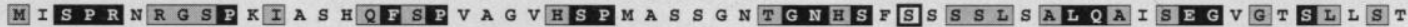

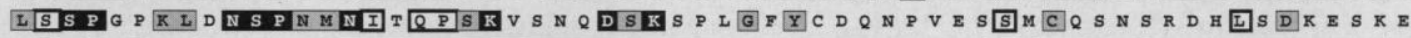

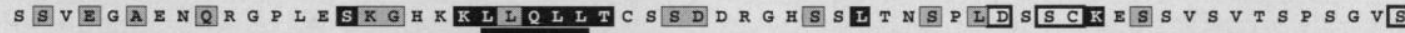

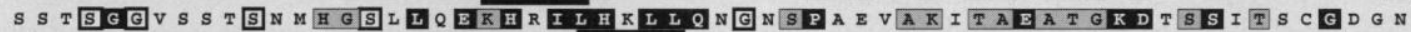

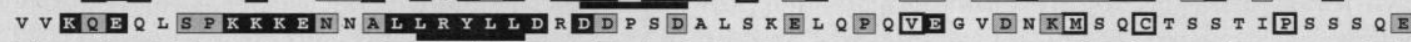

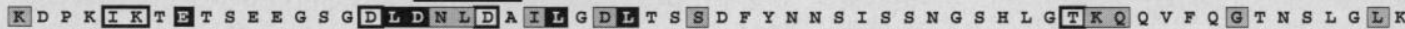

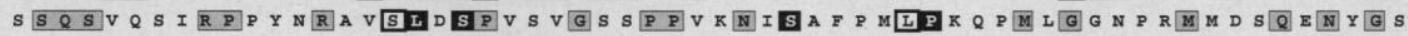

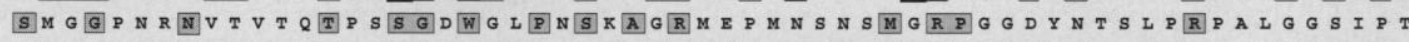

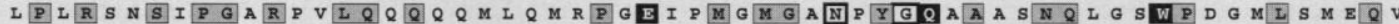

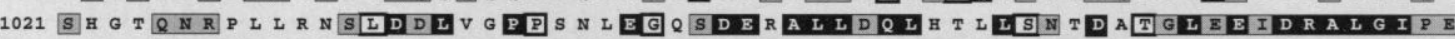
1081 LVN R 1141 回M NQQ 固N

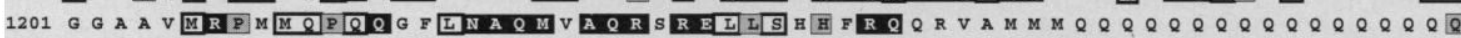

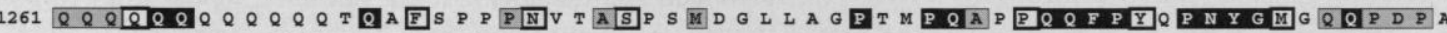

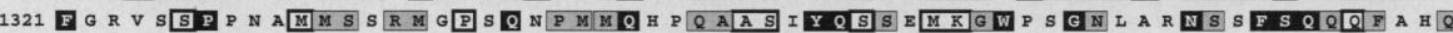
1381 G

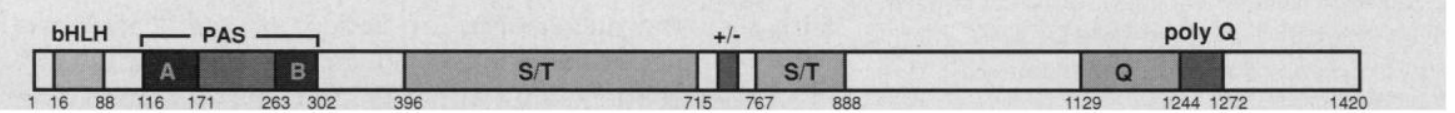


mechanisms other than amplification also occurs frequently in human breast cancers. ER status, as determined by immunohistochemistry, was not strongly correlated with either AIB1 amplification or expression in the unselected tumors $(12,15)$. However, both clinical specimens that demonstrated independent high-level AIB1 amplification were from postmenopausal patients and were ER and progesterone receptor-positive. One of these specimens was a metastasis from a patient who subsequently responded favorably to treatment with tamoxifen.

We then sought to determine whether expression of AIB1 increases ER liganddependent transactivation. To accomplish this, we performed transient transfection assays to examine the effect of increasing amounts of AIB1 on transcription of an ER-dependent reporter (16). These results conclusively demonstrated that cotransfection of AIB1 led to a dose-dependent increase in estrogen-dependent transcription

Fig. 3. (A) Increased expression of AlB1 in the AIB1 amplified cell lines BT-474, ZR-75-1, MCF7, and BG-1 is apparent on Northern analysis. The blot was hybridized sequentially with the indicated probe to compare AlB1 expression with that of ER, TIF2, and SRC-1. To avoid cross-hybridization between these related genes and to match signal intensities, we used similar-sized probes from the $3^{\prime}$ untranslated regions of AlB1, TIF2, and SRC-1 (23). Each of these probes detected a signal in normal mammary RNA (Clontech) on longer exposure. Electrophoresis, transfer, and hybridization of $15 \mu \mathrm{g}$ of total RNA was performed by standard methods (24). We used a $\beta$-actin probe as a control for loading error. (B) Dark-field microscopic image of AlB1 mRNA in situ expression demonstrating moderate expression in normal breast epithelium (left) and high-level expression of AIB1 in malignant mammary epithelial cells carrying increased copies of the AlB1 gene (right) with marked cell to cell heterogeneity.

Fig. 4. AlB1 increases estrogendependent transcription from an ER reporter plasmid in vivo and interacts with the ER in GST pulldown assays. (A) CV-1 cells were transiently transfected with $250 \mathrm{ng}$ of ER expression vector (pHEGOhyg), $1.0 \mu \mathrm{g}$ of luciferase reporter plasmid (pGL3.luc.3ERE or pGL3 lacking ERE), and increasing amounts of pCDNA3.1-AIB1 and in cubated in the absence (open bars) or presence of $10 \mathrm{nM} 17 \beta$-estradiol (E2) (solid bars) or $100 \mathrm{nM} 4 \mathrm{OHT}$ (hatched bars). Luciferase activity is

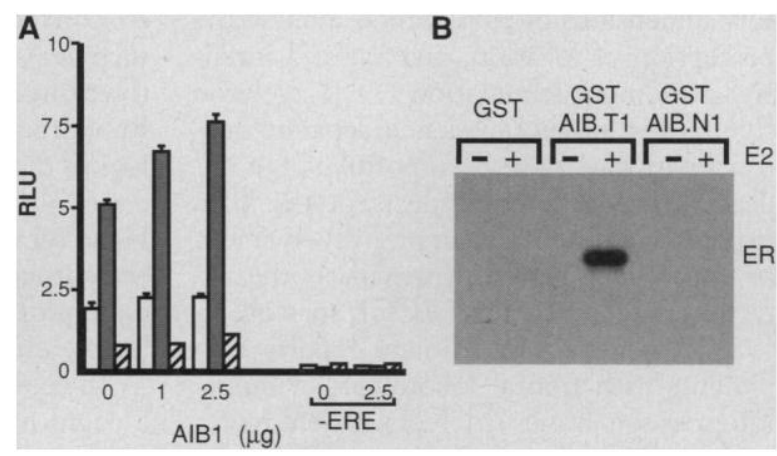

expressed in RLU. Data are means of three determinations from one of four replicate experiments. Error bars indicate 1 SD. (B) AIB1 protein binds to ER in a ligand-dependent fashion. To examine the interaction of ER with AIB1 in vitro, we incubated unliganded or estradiol-treated ER with glutathioneSepharose beads containing GST, GST-AIB.T1 (amino acids 605 to 1294), or GST-AIB.N1 (amino acids 1 to 194). Note that interaction is specific for the AlB1.T1 fragment that contains the LXXLL motifs. Bound ER was visualized by SDS-PAGE, immunoblotting, and chemiluminescence.

(Fig. 4A). This increase was not observed when the estrogen antagonist 4-hydroxytamoxifen (4-OHT) was substituted for $17 \beta$ estradiol or when the estrogen response element (ERE) was removed from the reporter plasmid (Fig. 4A). The degree of coactivation was not as pronounced as that reported SR-1 and TIF2, but it was comparable to that observed with the very recently described murine SRC-1 family member $\mathrm{p} / \mathrm{CIP}$ (10). Further evidence of ER-AIB1 interaction was provided by a glutathione $S$-transerase (GST) pulldown assay (17), which demonstrated that a GST fusion protein that contains the region predicted to contain the NR interaction domain (residues 605 to 1294 of AIB1) associates with ER in a ligand-dependent fashion (Fig. 4B). These results demonstrate that AIB1 interacts directly with ER and increases estrogen-dependent ranscriptional activity.

This study has identified AIB1 as member of the SRC-1 family of transcriptional coactivators that is frequently am-

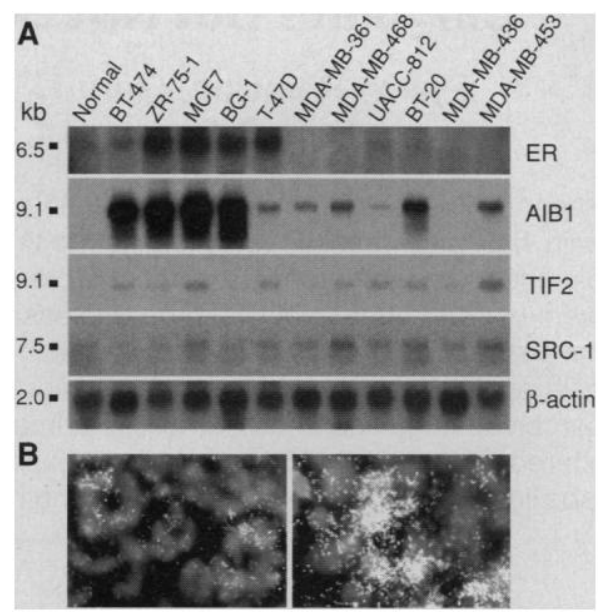

plified and overexpressed in breast cancer. Its amplification, effect on estrogen-dependent transcription, and interaction with ER implicate AIB1 as an important component of the estrogen-response pathway. On the basis of the observation that GRIP1, the mouse ortholog of TIF2, is a coactivator of multiple NRs (18) and the presence in AIB1 of the newly described LXXLL NR interaction motifs $(9,10)$, we do not expect that AIB1 coactivation is confined to ER alone. It is likely that ER is a major but not exclusive target of AIB1-NR interaction in mammary epithelial cells. Accumulation of excess quantities of the usually limiting AIB1 protein could have a profound effect on the expression of numerous genes that are normally regulated by NRs. Additionally, interaction with $\mathrm{CBP} / \mathrm{p} 300$ is important in the function of SRC-1, TIF2, and p/CIP; it also is likely to be important for AIB1 function $(6,10)$. Thus, AIB1 overexpression could potentially perturb signal integration by $\mathrm{CBP} / \mathrm{p} 300$ and affect multiple signal transduction pathways. Our observations of AIB1 amplification suggest that the resulting dysregulation of gene expression provides a selective advantage for tumor growth.

\section{REFERENCES AND NOTES}

1. P. Devilee, E. Schuuring, M. J. van de Vijver, C. J. Cornelisse, Crit. Rev. Oncog. 5, 247 (1994).

2. J. J. Isola et al., Am. J. Pathol. 147, 905 (1995); A. Kallioniemi et al. Proc. Natl. Acad Sci U.S.A. 91, 2156 (1994); M. Muleris, A. Almeida, M. GerbaultSeureau, B. Malfoy, B. Dutrillaux, Genes Chromosomes Cancer 10, 160 (1994); M. M. Tanner et al., Cancer Res. 54, 4257 (1994); X.-Y. Guan, P. S. Meltzer, W. S. Dalton, J. M. Trent, Nature Genet. 8, 155 (1994)

3. X.-Y. Guan et al., Cancer Res. 56, 3446 (1996).

4. S. A. Onate, S. Y. Tsai, M.-J. Tsai, B. W. O'Malley, Science 270, 1354 (1995)

5. J. J. Voegel, M. J. S. Heine, C. Zechel, P. Chambon, H. Gronemeyer, EMBO J. 15, 3667 (1996).

6. B. Hanstein et al., Proc. Natl. Acad. Sci. U.S.A. 93 11540 (1996); Y. Kamei et al., Cell 85, 403 (1996) T.-P. Yao, G. Ku, N. Zhou, R. Scully, D. M. Livingston, Proc. Natl. Acad. Sci. U.S.A. 93, 10626 (1996)

7. An AIB1-specific primer, N8F1 (TCATCACTTCCGACAACAGAGG), was biotinylated and used to capture cDNA clones from a human lung cDNA library (Gibco, BRL) with the GENETRAPPER CDNA positive selection system (Gibco, BRL). The largest clone (5.8 kb), designated PCMVSPORT-B11, was selected for sequence analysis. To obtain full-length AlB1 sequence, we constructed a random-primed library from BT474 in bacteriophage $\lambda$-Zap (Stratagene) and hybridized it with a 372 -base pair ${ }^{32} \mathrm{P}$ labeled polymerase chain reaction (PCR) product amplified from a human spleen cDNA library by using primers designed from the 5 ' sequence of pCMVSPORT-B11, PM-U2 (CCAGAAACGTCACTATCAAG), and BII-IIRA (TTACTGGAACCCCCATACC). Plasmid rescue of 19 positive clones yielded a clone, pBluescript-R22, that overlapped pCMVSPORT-B11 and contained the $5^{\prime}$ end of the coding region. To generate a full-length AIB1 clone, we subcloned the $4.85-\mathrm{kb}$ Hind III-Xho I fragment of PCMVSPORTB11 into the Hind III-Xho I sites of pBluescript-R22. We then subcloned the 4.84-kb Not I-Nhe I fragment of the full-length clone containing the entire 
coding region into the Not I-Xba I sites of the expression vector PCDNA3.1 (Invitrogen) and generated pcDNA3.1-AIB1.

8. S. F. Altschul, W. Gish, W. Miller, E. W. Myers, D. J. Lipman, J. Mol. Biol. 215, 403 (1990).

9. D. M. Heery, E. Kalkhoven, S. Hoare, M. G. Parker, Nature 387, 733 (1997)

10. J. Torchia, et al., Nature 387, 677 (1997).

11. We obtained established breast cancer cell lines from the American Type Culture Collection (ATCC) (BT474, MCF-7, T-47D, MDA-MB-361, MDA-MB 468, BT-20, MDA-MB-436, and MDA-MB-453), the Arizona Cancer Center (UACC-812), and the National Cancer Institute (ZR75-1). We obtained ovarian cancer cell line BG-1 from Jeff Boyd, University of Pennsylvania. For Northern analysis, we obtained normal human mammary gland total RNA pooled from six individuals between 16 and 35 years old from Clontech. For FISH analysis, interphase nuclei were fixed in methanol and acetic acid $(3: 1)$ and dropped onto microscope slides.

12. Data not shown.

13. The BioPrime DNA labeling system (Gibco BRL) was used to label genomic clones containing either AIB1 (3) or the 20q13 amplicon (RMC20C001) (14) with Spectrum Orange deoxyuridine triphosphate (dUTP) (Vysis). We used 20q11 and 20p probes (University of California, Berkeley, Resource for Molecular Cytogenetics RMC20P037 and RMC20P039) as reference probes for two-color FISH analysis after labeling with biotin-16-dUTP (BMB) by nick-translation. FISH followed the method of Pinkel et al. (19) with minor modifications. Fluorescent images were captured with a Zeiss axiophot microscope equipped with a charge-coupled device camera and IP Lab Spectrum software (Signal Analytics). FISH analysis of uncultured breast cancer samples was performed as described (14) from either disaggregated nuclei or sections of ethanol-fixed tumors. Tumors were categorized into three groups according to AIB1 copy number: low (fewer than four copies per cell or no increased relative copy number), moderate (four to six copies per cell or 1.5 - to 3 -fold relative copy number), and high (more than six copies per cell or $>3$-fold relative copy number). Only high copy number increases were taken as evidence for gene amplification. In 105 unselected cases, 10 were scored high, 13 were moderate, and 82 were low. ER status was determined by immunohistochemistry for 83 of these specimens including 9 of the 10 cases with AlB1 amplification. Of these nine specimens, four were ER positive and five were ER negative

14. M. M. Tanner et al., Cancer Res. 56, 3441 (1996)

15. AlB1 mRNA in situ expression was determined by using three cDNA fragments (covering nucleotides 1733 to 2579,3072 to 3580 , and 3533 to 4120 of the AIB1 cDNA) labeled with deoxyadenosine [ $\left.{ }^{33} \mathrm{P}\right] \mathrm{t}-$ riphosphate by PCR. We hybridized ethanol-fixed sections from 75 primary breast tumors and six adjacent normal breast tissues as described (20). We knew the ER status for 66 of these samples. AlB1 was highly expressed in 24 of $44(55 \%)$ of the ERpositive cases and in 8 of $22(36 \%)$ of the ER-negative cases.

16. CV-1 cells (ATCC) were grown and maintained in phenol red-free Dulbecco's modified Eagle's medium supplemented with $10 \%$ charcoal-stripped fetal bovine serum. We plated cells into six-well culture dishes at $1.0 \times 10^{5}$ cells per well and allowed them to grow overnight. Transfections were done with calcium phosphate coprecipitation (Clontech) according to the manufacturer's protocol. Transfected DNA included $5 \mathrm{ng}$ of $\mathrm{pRL}-\mathrm{CMV}$ internal control plasmid, $1.0 \mu \mathrm{g}$ of ER reporter, $250 \mathrm{ng}$ of pHEGO-hyg ER expression vector, the indicated amount of pcDNA3.1-AIB1, or an equivalent amount of pcDNA3.1 empty vector, and salmon sperm DNA to a total of $7 \mu \mathrm{g}$ of DNA per dish. After transfection, we incubated cells in the absence or presence of $10 \mathrm{nM} 17 \beta$-estradiol or $100 \mathrm{nM} \mathrm{4-}$ $\mathrm{OHT}$. Cell Iysates were harvested and assayed 48 hours after transfection. We determined reporter activities with the dual-luciferase reporter assay system (Promega) and the results are expressed in relative luminescence units (RLU) (luciferase/Renilla luciferase). We obtained PRL-CMV and pGL3-promoter

from Promega and pHEGO-hyg from ATCC. ER reporter pGL3.luc.3ERE, which contains three tandem copies of the ERE upstream from the simian virus 40 promoter driving the luciferase gene, was the kind gift of Fern Murdoch, Uniformed Services University of the Health Sciences.

17. We constructed GST fusion proteins by generating PCR fragments of AlB1 encoding amino acids 1 to 194 and amino acids 605 to 1294 inserted into pGEX 6P-2 (Pharmacia). GST pulldown analysis was done as described by Le Douarin (21) with the following modifications: $6.7 \mu \mathrm{g}$ of ER (Panvera) was preincubated with or without $2 \mu \mathrm{M}$ estradiol. Separately, we preincubated $10 \mu \mathrm{g}$ of either GST, GST-AlB.N1 (containing amino acids 1 to 194), or GST-AIB.T1 (containing amino acids 605 to 1294) with preequilibrated glutathione-Sepharose (Pharmacia) and washed it with binding buffer. ER with or without estradiol mixture was incubated with GST fusion protein-glutathione-Sepharose mixture in binding buffer containing $0.1 \%$ bovine serum albumin for 1 hour at room temperature. Glutathione-Sepharose beads were washed five times in binding buffer, and bound proteins were eluted in SDS-polyacrylamide gel electrophoresis (SDS-PAGE) sample buffer and separated by SDS-PAGE. Separated proteins were transferred to nylon membranes, incubated sequentially with monoclonal antibody $\mathrm{H}-151$ to ER (StressGen) and horseradish peroxidase containing goat

\title{
Pain Affect Encoded in Human Anterior Cingulate But Not Somatosensory Cortex
}

\author{
Pierre Rainville, Gary H. Duncan, Donald D. Price, \\ Benoît Carrier, M. Catherine Bushnell ${ }^{*}$
}

Recent evidence demonstrating multiple regions of human cerebral cortex activated by pain has prompted speculation about their individual contributions to this complex experience. To differentiate cortical areas involved in pain affect, hypnotic suggestions were used to alter selectively the unpleasantness of noxious stimuli, without changing the perceived intensity. Positron emission tomography revealed significant changes in pain-evoked activity within anterior cingulate cortex, consistent with the encoding of perceived unpleasantness, whereas primary somatosensory cortex activation was unaltered. These findings provide direct experimental evidence in humans linking frontallobe limbic activity with pain affect, as originally suggested by early clinical lesion studies.

Affective aspects of pain, such as perceived unpleasantness, have been classically considered to be distinct from the simple sensory dimensions of pain, which include the perception of location, quality, and intensity of noxious stimulation (1). Largely on the basis of indirect evidence, separate neuronal pathways have been postulated to underlie these different aspects of the pain experience (2). For example, involvement of frontal lobe regions, particularly the anterior cingulate cortex (ACC), in pain affect is suggested by clinical reports that patients with frontal lobotomies or cingulotomies sometimes still feel pain but report it as less distressing or bothersome (3). On the other hand, primary and secondary somatosensory cortices (SI and SII) have been considered plausible candidates for the processing of sensory-discriminative aspects of pain, on the basis of their anatomical connections to subcortical and spinal re- gions, which encode discriminative properties of somatosensory stimuli (4). Recent neuroimaging studies in humans documented pain-related activation in limbic sites, such as ACC and rostral insula (IC), and in the primary sensory regions SI and SII (5). In addition, anatomical and electrophysiological data show that these regions receive direct nociceptive input in the monkey (6). However, the extent to which these different cortical structures contribute to specific dimensions of the human pain experience is largely unknown and untested.

In the present study we used hypnosis as a cognitive tool to reveal possible cerebral mechanisms of pain affect in normal human volunteers. A perceptual dissociation of sensory and affective aspects of the pain experience was achieved with hypnotic suggestions to both increase and decrease pain unpleasantness, without changing the perceived intensity of the pain sensations (7). 